\title{
Back to Woodworth: Role of interlopers in the tip-of-the-tongue phenomenon
}

\author{
GREGORY V. JONES \\ University of Warwick, Coventry, England
}

\begin{abstract}
When a person reports that a word is on the tip of his or her tongue, that person often recalls instead another word that is similar in sound to the target word. Two opposite roles have been suggested for these interlopers. An older view (Woodworth, 1929) holds that they are instrumental in the development of tip-of-the-tongue (TOT) states because they obstruct successful retrieval of intended targets. A more recent view (R. Brown \& McNeill, 1966) holds, on the other hand, that interlopers tend to nullify TOT states by facilitating complete retrieval of the intended tar. gets. A study is reported in which participants were explicitly presented with interloper words. The results provide two planks of support for Woodworth's hypothesis. First, more TOT states occurred when the interloper was similar in sound to the target than when it was not. Second, more TOT states occurred when the interloper was presented at the actual time of retrieval than when it was presented earlier. It appears that interlopers tend to induce TOT states by obstructing retrieval, rather than to nullify them by facilitating retrieval.
\end{abstract}

Few textbooks of cognitive psychology, or even of psychology tout court, have failed in recent years to describe the work on the tip-of-the-tongue (TOT) experience that was reported in 1966 by $R$. Brown and McNeill. The fame of this work is well justified. R. Brown and McNeill showed that the TOT experience, hitherto a feral beast that struck without warning, could be domesticated and studied in the laboratory as a creature of conveniently regular habits. As a result, they were able to confirm and extend a range of observations previously made outside the laboratory, for example, Woodworth's (1929) observation that "the initial sound of the true name is likely to be present in the recalled name, though not always in the first position. The number of syllables and the accent are usually preserved" (p. 100).

In view of the fact that there is a considerable degree of continuity between previous empirical observations and those of R. Brown and McNeill (1966), it is perhaps surprising to note that $R$. Brown and McNeill's theoretical interpretation of the observations differed fundamentally from the prevailing orthodoxy. The crucial finding is the frequent production in TOT states of a word that is similar in sound to the one that is being sought. For example, someone seeking the word sextant might produce instead the word secant. What is the significance of this observation? Woodworth had long suggested, on the basis of

The author is grateful to Deborah Burke, Gary Dell, Trevor Harley, Margaret Jean Intons-Peterson, David McNeill, Henry Roediger, three anonymous reviewers, and members of the 1987 Summer School on Cognitive Approaches to Memory (held by the European Society for Cognitive Psychology at Starnberger See, Federal Republic of Germany) for helpful discussions and comments. Correspondence concerning this article should be addressed to Gregory V. Jones, Department of Psychology, University of Warwick, Coventry CV4 7AL, England. his own and other corpus studies (see Woodworth, 1938), that the interloping word promotes the TOT experience by heading off retrieval of the sought-after word (e.g., Woodworth, 1929; Woodworth \& Marquis, 1947). R. Brown and McNeill, on the other hand, proposed that the effect of the interloper on retrieval is opposite in nature, facilitating rather than disrupting retrieval. As an example, they proposed that a TOT state might occur with a lexical entry of the form SextanT, where lowercase letters are only faintly entered. The occurrence of an interloper such as secant is hypothesized to allow the overlapping letters of the target to be rendered accessible (yielding SExtANT), in a manner similar to "the way heat brings out anything written in lemon juice," as R. Brown and McNeill (1966, p. 335) colorfully expressed it. According to this view, therefore, the interloping words are instrumental in nullifing rather than promoting the TOT state.

R. Brown and McNeill did not themselves point to any difference between their own and previous interpretations of the role of similar-sounding words in the TOT state. Nor does it seem to have been noted by others (e.g., Koriat \& Lieblich, 1974; Reason \& Lucas, 1984; Rubin, 1975) who have subsequently investigated the TOT phenomenon. Nevertheless, the difference between the two theories is in principle eminently open to experimental investigation by methods that represent only a small development of those introduced by $\mathrm{R}$. Brown and McNeill. If a person is explicitly provided with a word that is similar in sound to a target word that he or she attempts to retrieve, two alternative predictions can be assessed. The Woodworth view suggests that the artificial provision of such an interloper will lead to an increase in the likelihood of a TOT state's being reported, whereas the BrownMcNeill view suggests a decrease in this likelihood. 


\section{TESTING THE WOODWORTH AND BROWN-MCNEILL PREDICTIONS}

In the experiment reported here, participants were presented with definitions of moderately rare words, and attempted to retrieve these target words from memory. The first focus of this study was upon the fact that each definition was accompanied by an additional word that might or might not be a neighbor of the target word in either sound or meaning. The Woodworth and BrownMcNeill views predict that the presence of interlopers that are neighbors in sound should give rise, respectively, to increased or to decreased incidences of TOT states. Interlopers of some type were thus used in all the experimental conditions, because the presence even of a completely unrelated word as interloper may have some effect upon the incidence of TOT states. If this is so, it would be inappropriate when investigating the effect of similarity in sound between target and interloper to use as a baseline condition one in which no interloper at all is present.

Although they did not contrast the Woodworth and Brown-McNeill hypotheses in the way undertaken here, Jones and Langford (1987) recently reported some data that bear on the point at issue. They found that the presence of an interloper similar in sound to the word that was sought did lead to an increase in the number of reported TOT states, providing prima facie evidence in favor of the Woodworth hypothesis. Further work is desirable for several reasons, however. First, participants in Jones and Langford's study had to record only the presence of a TOT state and not necessarily any further information. To encourage the adoption of a more uniform strict criterion, participants in the present experiment were requested to estimate characteristics of the target word whenever in a TOT state, as were subjects in the original experiment of $\mathbf{R}$. Brown and McNeill. Second, similarity in sound between the target and the additional word was defined by Jones and Langford in terms of initial letter overlap rather than initial phoneme overlap. In the present experiment, the stimuli were matched on both letters and phonemes. Third, the present experiment provided an opportunity to assess how generalizable the effects of interlopers are.

The second focus of the study was upon the temporal pattern of activation of target and interloper word representations. If interlopers are to exert either a positive (Woodworth) or a negative (Brown-McNeill) effect on the incidence of TOT states, their mental representations presumably need to be activated at approximately the same time as retrieval of the corresponding target representations is attempted. Since the retrieval of a target, of course, follows the presentation of its definition, it is to be expected that the maximum increase (Woodworth) or decrease (Brown-McNeill) in TOT states will occur when the interloper occurs at this very point-that is, directly following the definition. Half of the participants in the present experiment did indeed receive interlopers as suffixes to the definitions. The other half received prefix interlopers, that is, interlopers whose presentation occurred before that of their corresponding definitions commenced. The Woodworth hypothesis thus predicts that a greater incidence of TOT states will occur with suffix interlopers than with prefixes, whereas the Brown-McNeill hypothesis predicts that a smaller incidence of TOT states will occur with suffixes than with prefixes.

\section{EXPERIMENT}

\section{Method}

Subjects. The subjects of this experiment were 116 undergraduates studying Stage I Psychology at Bristol University.

Design. Each subject was presented with the same set of 40 definitions of target words. Each definition was accompanied by an additional word that served as an interloper. All 40 target words and 40 interlopers were selected from the 9,202 words listed by Thorndike and Lorge (1944) as occurring at a rate of less than one per million, but at least one per 4 million. Examples of targets and their definitions are as follows: Anachronism-Something out of keeping with the times in which it exists. Braise-To steam food, particularly meat, slowly in a closed container. Banshee-Female spirit whose wail portends death. Hospice-House of rest for travelers or for the terminally ill, often kept by a religious order. The interlopers fell into four categories of 10 words each. They were related in both sound and meaning to targets (e.g., abnormality for the target anachronism), related only in sound (e.g., baulk for braise), related only in meaning (e.g., incubus for banshee), or related in neither sound nor meaning (e.g., fossilize for hospice). A complete list of definitions, interlopers, and targets is given in the Appendix.

Words that were categorized as related in sound had the same initial letter and phoneme, and the same number of syllables, whereas words not related in sound had different values in each case. Categorization of words as either related in meaning or not related in meaning was originally made on the basis of experimenter judgment. The reliability of this categorization was confirmed in a subsidiary experiment, in which 41 undergraduates studying second-year psychology at Warwick University judged on a scale from 1 to 4 how strongly related in meaning they thought each of the target-interloper pairs of words were. It was found that the average judgment for words categorized as related in meaning (2.61, $S D=0.46$ ) was much higher than that for words categorized as not related in meaning $(1.32, S D=0.32)[t(40)=20.79$, $p<.001]$.

The 40 stimuli used in the present experiment were obtained by discarding 20 of the 60 stimuli used previously by Jones and Langford (1987). A stimulus was discarded if its target and interloper matched in initial letter but not in initial phoneme, if one of the two words seemed particularly obscure (e.g., dispart), or if the definition seemed relatively imprecise (e.g., impolite-ill-mannered, acting without consideration or tact). Stimuli were discarded in ignorance of their associated levels of performance in the study of Jones and Langford (1987).

All subjects were presented with the $\mathbf{4 0}$ stimuli in the same order. In each case, the definition and the interloper were spoken once immediately after the other by the experimenter, who rapped the desk between the two utterances to signal their demarcation. For half of the subjects each definition preceded its interloper, whereas for the other half this order was reversed. The presentation of each stimulus (i.e., definition and interloper) was repeated after a delay of approximately $4 \mathrm{sec}$, so as to ensure full comprehension.

Procedure. The stimuli were presented in an order balanced over the four categories of interloper (similar/dissimilar in sound/meaning 
with respect to the target). Each successive set of four stimuli contained one member of each category, and the average serial position for each category was the same. Furthermore, there were never more than two stimuli in succession sharing a common attribute (e.g., similarity in sound between interloper and target), and the initial letter of a target was never repeated in adjacent stimuli.

The instructions for the experiment described to the subjects what a TOT state is and explained that it might occur following the pro vision of a word's definition. The subjects were also told that they would receive interlopers that were not themselves targets but that might be related to the targets; however, the subjects were not told how the interlopers might be related to the targets. Before each stimulus was presented, the experimenter announced its serial position (1 to 40). Each subject had a response form with sections numbered from 1 to 40 . Following the presentation of each stimulus, there was a response period of approximately $30 \mathrm{sec}$ before the next trial commenced. If a TOT state occurred during this time the subject was instructed to enter a best estimate of the target's attributes in each of two parts of the relevant section, headed "Initial letter" and "Number of syllables."

\section{Results}

Altogether, 457 TOT states were recorded. The initial letter of the target word was estimated correctly in $41.6 \%$ of the cases (190 out of 457), and the number of syllables in $33.9 \%$ of the cases (155 out of 457 ).

Table 1 shows the total number of TOT states occurring for each experimental condition. Analysis of variance was carried out on the data for each subject and stimulus. When both subjects and stimuli were treated as random effects (the procedure argued for by Clark, 1973), it was found that there were two significant $(p<.05)$ effects. More TOT cases occurred when the interloper was related in sound to the target $(62.1 \%)$ than when it was not $(37.9 \%)\left[\min F^{\prime}(1,50)=5.74\right]$, and more TOT cases occurred when the interloper was a suffix $(59.1 \%)$ than when it was a prefix $\left(\mathbf{4 0 . 9 \% )}\left[\min F^{\prime}(1,146)=\right.\right.$ 3.95]. There was no significant effect of whether or not the interloper was related in meaning to the target, and no significant interaction. Identical patterns of significance were found if either subjects or stimuli were treated separately as random effects, again with no significant effects of relatedness in meaning or of any interaction. Thus, if only subjects were treated as random effects, there were again only the same two significant effects, those of related/not related in sound $[F(1,114)=36.64]$ and of prefix/suffix $[F(1,114)=4.76]$. Similarly, if only items were treated as random effects, there were once again only the same two significant effects of related/not related in

Table 1

Frequency of Tip-of-the-Tongue States for Each Experimental Condition

\begin{tabular}{lcccc}
\hline Interloper & \multicolumn{4}{c}{ Interloper-Target Relation } \\
\cline { 2 - 5 } Position & S,M & S,NM & NS,M & NS, NM \\
\hline Prefix & 65 & 64 & 30 & 28 \\
Suffix & 73 & 82 & 57 & 58 \\
\hline
\end{tabular}

Note-S = related in sound. $M=$ related in meaning. $N S=$ not related in sound. $\mathrm{NM}=$ not related in meaning. sound $[F(1,36)=6.80]$ and of prefix/suffix $[F(1,36)=$ 23.37].

\section{Discussion}

The rate of occurrence of TOT states was a little lower in tine present experiment $(9.8 \%$ of all trials; 457 out of $4,640)$ than in that of R. Brown and McNeill $(13.1 \%$ of all trials; 360 out of 2,744 ), possibly owing to subject and item differences between the studies. For example, targets in the present experiment possessed more syllables (mean $=3.2$ ) than those in R. Brown and McNeill's experiment (mean $=2.4$ ).

The lengths of the target words in the present experiment may well have been partly responsible for the relatively low level $(33.9 \%)$ of correct estimation of the target words' numbers of syllables. How does this level compare with the performance that could be achieved simply by guessing? One guessing strategy would be to respond always with the modal number of syllables per word of the word sample (if this mode can be correctly inferred). Since there were $1,10,14,9$, and 6 targets of lengths $1,2,3,4$, and 5 syllables, respectively, this strategy would yield an expected guessing level (as a result of always responding "three") of $35 \%$ (i.e., 14/40), numerically higher than the observed level of performance. An alternative to a modal guessing strategy would be a probability-matching strategy, in which responses are distributed so as to mirror the stochastic distribution of syllable lengths within the word sample (again, assuming that the latter distribution can be correctly inferred). This strategy would yield a somewhat lower guessing level for correct estimation of $\Sigma(n / 40)^{2}$, or $25.9 \%$. It is thus apparent that the present experiment provides rather little evidence that the presence of a TOT state leads to correct estimation, beyond the chance level, of a target's number of syllables.

In their experiment, R. Brown and McNeill (1966) did not report the accuracy of estimation overall, but instead excluded cases in which subjects subsequently were unable to definitely indicate that their TOT states had been directed toward known target words. For their remaining $64.7 \%$ of "positive" cases (233 out of 360 ), the level of correct estimation of number of syllables was $56.7 \%$ (132 out of 233) overall, but was only $23.3 \%$ (7 out of 30) for targets of more than 3 syllables. It may also be noted that the modal guessing strategy (in this case, again always guessing "three") would have been successful overall on $42.5 \%$ of occasions (99 out of 233 ; the ratio refers here not directly to R. Brown and McNeill's intended 49 target words, but rather to the ratio for all 233 positive TOT states, with the number of syllables of any subject-supplied targets substituted where appropriate). Similarly, the probability-matching strategy would have been successful on $33.7 \%$ of occasions $(17,87,99,25$, and 5 targets of $1,2,3,4$, and 5 syllables, respectively). Thus, even with $R$. Brown and McNeill's generally 
shorter words, the level of correct estimation of syllable length when in a TOT state occurred at only a moderate increment above possible chance levels.

Accuracy of identification of a word's initial letter when in a TOT state in the present experiment was, in contrast to estimation of number of syllables, well above chance levels at $41.6 \%$. Even the modal guessing strategy (in this case, always guessing " $b$ " as the initial letter) would have achieved an expected level of correct estimation of only $15 \%$ (6 out of 40). Similarly, R. Brown and McNeill observed a level of correct identification of initial letters in their positive TOT cases that was much above chance levels at $50.2 \%$ (the figure of $57 \%$ is given on p. 329 of R. Brown \& McNeill, 1966, but this is contradicted by the data reported on p. 332 and perhaps resulted from confusion with the figure for number of syllables).

An important finding in the present experiment was the observation that interlopers induced more TOT states when presented as suffixes to definitions than when presented as prefixes to them. The fact that presentation of the interloper at the time of recall led to more TOT states than did presentation at an earlier stage reinforces the conclusion that the effect of the interloper upon recall is to hinder it, as proposed by Woodworth, rather than to help it, as proposed by R. Brown and McNeill. At this point, however, we may consider also a possible objection to the use of the prefix/suffix manipulation. The objection would be that the difference in time between prefix and suffix is not sufficiently large for forgetting of the interloper to be significantly greater in the former condition, and indeed that the practice of presenting each stimulus twice would have tended to neutralize this contrast further. It is argued here, however, that this objection may be discounted for two reasons. The first is that forgetting of the interloper seems in fact unlikely to be the relevant factor in comparing the two stimulus conditions. Rather, the level of activation of the interloper's representation seems the more salient parameter, and also one whose magnitude may indeed change considerably over the short term. The second reason for discounting the objection is empirical rather than theoretical. The a priori argument that there is no reason to expect a difference between prefix and suffix conditions was rebutted here by the fact that such a difference nevertheless was observed in practice.

Further implications of this study are discussed in the following section.

\section{GENERAL DISCUSSION}

The present experiment was carried out to provide evidence that would help to discriminate between two different theories of the role played by the near-miss words frequently generated by people undergoing TOT episodes. Woodworth (1929) hypothesized that these interlopers are instrumental in the causation of the TOT state, whereas R. Brown and McNeill (1966) hypothesized that they are instrumental in its erasure. The results of the experiment provided clear support for the Woodworth hypothesis. First, they confirm that the provision of an interloper similar in sound to the target word leads to the observation of more rather than fewer TOT states, and show that this finding may be generalized over both subjects and words. Second, the results demonstrate that an increase in the number of TOT states occurs when the interlopers are presented at the actual time of retrieval (rather than earlier), again suggesting that the effect of these interlopers is to disrupt retrieval and hence encourage the formation of TOT states. The latter effect did not interact statistically with the effect of similarity in sound between interloper and target. This result implies, therefore, that even the occurrence of an interloper that is not similar in sound to the target may exert some effect on retrieval, albeit an effect that is less powerful than that of an interloper that is similar in sound to the target.

Although the evidence reported here indicates that interlopers similar in sound to target words act to increase the incidence of TOT states as hypothesized by Woodworth, rather than to decrease it as hypothesized by R. Brown and McNeill, it should be noted that consideration may also be given to another possibility. It could be that interlopers do, in fact, exert an effect of the second kind, but that this effect is small relative to the effect of the first kind in the opposite direction. Perhaps the most direct way of examining this possibility would be to modify the present procedure as follows: First, definitions are presented without explicit interlopers, as in R. Brown and McNeill's original study. Second, when a TOT state does occur, an interloper similar in sound to the target is (or is not) presented and its effect observed. In this situation, the causative role of the interloper in TOT production is rendered redundant, and thus any further, smaller role that the interloper might play in TOT erasure should be directly observable.

Let us consider further the successful termination of TOT states. Since R. Brown and McNeill (1966) offered the putatively facilitative effect of near-miss words on retrieval as an explanation of why the TOT state is often abbreviated by successful recall, it is appropriate to consider alternative explanations of this variability in the outcome of retrieval. One approach, of course, would be simply to credit recall with possessing an inherently random element, so that the outcomes of successive retrieval attempts fail to be perfectly correlated (e.g., as in the schema model examined by Jones, 1984). The Woodworth hypothesis suggests an additional alternative, however. If the TOT experience occurs when the retrieval of a target word is stymied by the generation (or experimental presentation) of some other word, then the absence of this interloper (and decay in the activation of its mental representative) should leave retrieval of the target word able to progress unimpeded. In particular, focusing on different aspects of the initial retrieval information might lead to the previous interloper's no longer receiving fresh activation, allowing recall of the target word to proceed to completion without further hindrance. 
The facilitative hypothesis of interloper function proposed by R. Brown and McNeill (1966) appears, therefore, not only to lack empirical support, but also to have no explanatory advantage over the earlier hypothesis of Woodworth.

\section{Implications for Theories of Lexical Retrieval}

Jones and Langford (1987) hypothesized that, in considering the TOT state, two successive stages of retrieval need to be distinguished. In the first, partial retrieval yields a word sketch that represents only the most important phonological features of a word, such as its initial phoneme and its number of syllables. In the second, these features themselves are used in retrieval of the word's complete specification. The sketch's features may match not only the target word, but also another similar-sounding word (such as a phonological interloper in the present study or a similar-sounding word in the everyday environment), which can therefore act as a decoy to deflect retrieval away from the intended target.

Can the consideration of other, related work cast further light on the retrieval mechanisms responsible for the phonological blocking effect? This question may be approached from at least two different directions, the first being that of work on memory retrieval and the second that of work on speech production.

Memory retrieval. From the perspective of work on memory retrieval, the present observations may be related to a number of other phenomena. For example, the generation effect (Slamecka \& Graf, 1978) refers to the finding that words are recalled better if previously generated actively in response to a cue (e.g., "What word is a synonym of rapid and starts with $f$ ?") rather than simply read passively (in this case, fast). Slamecka and Fevreiski (1983) found that this advantage survived even if the attempt to generate the word's name was unsuccessful. They attributed this facilitation in the presence of overall failure to the successful occurrence of an initial process of generation of semantic attributes, even though these themselves subsequently failed to access the desired phonemic information. Recent work has shown, however, that the generation advantage appears to occur only when generation and reading take place in the same list, and not when they take place in two separate lists (Begg \& Snider, 1987; Slamecka \& Katsaiti, 1987). This finding suggests that the occurrence of a generation effect within a mixed-item list is merely the consequence of limited attentional resources being directed away from read items and toward generated items.

More directly relevant to the present observations are a number of other instances in which completion of the retrieval process is blocked by other material (for a review, see Roediger \& Neely, 1982). Two phenomena that appear especially relevant are those of part-list cuing inhibition and of semantic priming inhibition. In the former, the provision of part of a target set of items is found to lead to impaired recall of the remainder of the items (J. Brown, 1968; Slamecka, 1968). Principled ex- planation of this phenomenon has proved to be quite difficult to achieve (see Roediger \& Neely, 1982), but one account may be given within the terms of the search of associative memory (SAM) model of Raaijmakers and Shiffrin (1981). According to SAM, in the absence of partlist cues, those items with the strongest context-to-item links are the ones that are retrieved initially, and go on to be relatively successful at instigating further recall because they possess also the strongest item-to-item links. In the presence of part-list cues, on the other hand, selection with respect to link strength does not occur and the retrieval process overall is weakened. Furthermore, because search in retrieval is hypothesized to be clustered, part-list cuing is also prejudicial because it ensures that one member of a retrieved cluster (the cue itself) is not an admissible target item.

At first sight, the semantic priming inhibition paradigm perhaps appears particularly closely related to the present investigation. In this paradigm (A. S. Brown, 1979), people are shown a word as prime, followed by a definition. They have to decide whether or not the definition refers to the prime word and, in the case that it does not, to attempt to generate the actual word to which the definition does refer. As an example, the correct prime gobble was followed by the definition "To swallow up or eat greedily." What A. S. Brown found was that, relative to a prime (in this case, feud) that was unrelated to the target word, the speed and accuracy of generation of the target were improved for a prime (in this case, goggle) related in sound to the target but were impaired for a prime (in this case, $\mathrm{cram}$ ) related in meaning. This dissociation between phonological and semantic effects bears some similarity to that observed in the present study, but is reversed in polarity. In Brown's study a phonological prime promoted successful recall, whereas in the present study a phonological interloper promoted instances of unsuccessful recall, in the shape of occurrences of the TOT state. Furthermore, the implications of Brown's findings are heavily constrained by the results of a recent study by Roediger, Neely, and Blaxton (1983). Roediger et al. found that the semantic inhibition effect disappears if all correct primes are removed from the experimental procedure, and concluded therefore that the effect is not the product of some fundamental pattern of lexical activation and inhibition in priming. Instead, the semantic inhibition effect appears to be simply the consequence of the relatively high level of difficulty involved in making the earlier decision as to whether or not a semantic prime is itself a correct target, in an experiment in which this is a procedural possibility. In the study reported here, on the other hand, no interloper could ever be a correct target, so this kind of factor could not have been operative.

Speech production. From the perspective of speech production, the TOT state may be considered as one of a number of different types of speech error. As summarized by Dell (1986, p. 312), speech errors as a general category tend to share the TOT characteristic of preferentially involving the initial sound of a word. Indeed, as 
summarized by R. Brown and McNeill (1966, p. 336), similar phenomena are widespread in other forms of verbal activity, such as reading and spelling. Unfortunately, however, the spreading-activation model for speech errors proposed by Dell possesses (as he himself noted) no inherent reason for expecting preferential involvement of initial sounds. How might the model interpret the phonological blocking observed in the present experiment? It distinguishes separate but highly interactive semantic, syntactic, morphological, and phonological levels of representation of an intended utterance, and asserts that

The closeness in the connection between similar items acts as a pathway for the activation of similar items to affect one another. An activated correct item will have similar competitors because of spreading, and if there are other factors that contribute to the activation of one of these competitors, the competitor's activation level may exceed that of the correct item leading to a slip. (Dell, 1986, p. 293)

The model thus appears to predict that semantic as well as phonological interlopers may induce TOT states, but presumably could attribute the empirical preponderance of the latter to competition in the TOT case being most intense at the phonological level.

A similar interpretation can be provided by interactive models of speech production, which propose that inhibition occurs among related nodes within the same level of representation, whereas facilitation occurs among related nodes within different levels (e.g., Harley, 1984; Stemberger, 1985; see also McClelland \& Rumelhart, 1981). Because the TOT state is characterized by difficulty in retrieving a target word's phonological representation, it is expected therefore to be especially likely to occur as a result of inhibition emanating from other phonologically related sources.

\section{REFERENCES}

BeGG, I., \& SNIDER, A. (1987). The generation effect: Evidence for generalized inhibition. Joumal of Experimental Psychology: Leaming, Memory, \& Cognition, 13, 553-563.

Brown, A. S. (1979). Priming effects in semantic memory retrieval processes. Journal of Experimental Psychology: Human Learning \& Memory, 5, 65-77.

BROWN, J. (1968). Reciprocal facilitation and impairment of free recall. Psychonomic Science, 10, 41-42.

Brown, R., \& McNeILL, D. (1966). The "tip of the tongue" phenomenon. Journal of Verbal Learning \& Verbal Behavior, 5, 325-337.
Clark, H. H. (1973). The language-as-fixed-effect fallacy: A critique of language statistics in psychological research. Journal of Verbal Learning \& Verbal Behavior, 12, 335-359.

DELL, G. S. (1986). A spreading-activation theory of retrieval in sentence production. Psychological Review, 93, 283-321.

HARLEY, T. A. (1984). A critique of top-down independent levels models of speech production: Evidence from non-plan-internal speech errors. Cognitive Science, 8, 191-219.

JoNES, G. V. (1984). Fragment and schema models for recall. Memory \& Cognition, 12, 250-263.

Jones, G. V., LANGForD, S. (1987). Phonological blocking in the tip of the tongue state. Cognition, 25, 115-122.

Koriat, A., \& Lieblich, I. (1974). What does a person in a "TOT" state know that a person in a "don't know" state doesn't know. Memory \& Cognition, 2, 647-655.

McClelland, J. L., \& Rumelhart, D. E. (1981). An interactive activation model of context effects in letter perception: Part 1. An account of basic findings. Psychological Review, 88, 375-407.

RaAijmakers, J. G. W., Shiffrin, R. M. (1981). Search of associative memory. Psychological Review, 88, 93-134.

Reason, J., Lucas, D. (1984). Using cognitive diaries to investigate naturally occurring memory blocks. In J. E. Harris \& P. E. Morris (Eds.), Everyday memory, actions and absent-mindedness (pp. 5370). Orlando, FL: Academic Press.

RoEdiger, H. L., \& NeEly, J. H. (1982). Retrieval blocks in episodic and semantic memory. Canadian Journal of Psychology, 36, 213242.

Roediger, H. L., NeEly, J. H., \& BlAXton, T. A. (1983). Inhibition from related primes in semantic memory retrieval: A reappraisal of Brown's (1979) paradigm. Joumal of Experimental Psychology: Leaming, Memory, \& Cognition, 9, 478-485.

Rubin, D. C. (1975). Within word structure in the tip-of-the-tongue phenomenon. Journal of Verbal Learning \& Verbal Behavior, 14, 392-397.

SLAMECKA, N. J. (1968). An examination of trace storage in free recall. Joumal of Experimental Psychology, 76, 504-513.

Slamecka, N. J., \& Fevreisku, J. (1983). The generation effect when generation fails. Journal of Verbal Leaming \& Verbal Behavior, 22, 153-163.

Slamecka, N. J., \& Graf, P. (1978). The generation effect: Delineation of a phenomenon. Journal of Experimental Psychology: Human Learning \& Memory, 4, 592-604.

Slamecka, N. J., KATsaiti, L. T. (1987). The generation effect as an artifact of selective displaced rehearsal. Joumal of Memory \& Language, 26, 589-607.

STEMBERGER, J. P. (1985). An interactive activation model of language production. In A. W. Ellis (Ed.), Progress in the psychology of language (Vol 1, pp. 143-186). London: Erlbaum.

THORNDIKE, E. L., LORGE, I. (1944). The teacher's word book of 30,000 words. New York: Teacher's College Press.

Woodworth, R. S. (1929). Psychology (2nd rev. ed.). New York: Holt.

WOODWORTH, R. S. (1938). Experimental psychology. New York: Holt.

WoOdWorth, R. S., MARQuIs, D. G. (1947). Psychology. New York: Holt.

\section{APPENDIX}

Definitions, Interlopers, and Targets for the Four Types of Interloper

\begin{tabular}{|c|c|c|}
\hline Definition & Interloper & Target \\
\hline \multicolumn{3}{|c|}{ Phonologically and Semantically Related Interlopers } \\
\hline $\begin{array}{l}\text { Something out of keeping with the times } \\
\text { in which it exists. }\end{array}$ & Abnormality & Anachronism \\
\hline To stupefy, bewilder, or confuse. & Bemock & Bemuse \\
\hline Of, on, or with two sides. & Binomial & Bilateral \\
\hline Someone who fails to act, to pay, or to & Disclaimer & Defaulter \\
\hline
\end{tabular}

appear for judgment in court. 
APPENDIX (Continued)

\begin{tabular}{lll}
\hline \multicolumn{1}{c}{ Definition } & Interloper & Target \\
\hline $\begin{array}{l}\text { State of mind in which one takes or } \\
\text { treats things lightly or with a }\end{array}$ & Fervency & Flippancy \\
lack of respect. & & \\
A young goose. & Gelding & $\begin{array}{l}\text { Gosling } \\
\text { The escape of blood from vessels, }\end{array}$ \\
Hepatic & Haemorrhage
\end{tabular}

including internal as well as external bleeding.

Descriptive term for the type of plant of Hibiscus Herbacious which the stem is not woody or persistent, and whose leaves and roots are often used for food, medicine, or scent.

Term used to describe a decision or person which is wicked or grossly unfair.

Able to read and write.

$$
\text { or }
$$

Gelding
Hepatic

Haemorrhage

Phonologically Related Interlopers

Sparing, moderate, not self-indulgent, especially in food and drink.

Medieval forerunner of chemistry.

Lover of books.

To steam food, particularly meat, slowly in a closed container.

Long close garment worn particularly by clergy and choristers, often under a surplice.

Adherent to the view that human action is not free but directed by external forces acting on the will.

A musical term for a passage performed with gradual decrease in loudness.

A person unnecessarily anxious about their health.

Referring to a phrase or way of expression natural or peculiar to a particular language.

Game like hockey but with ball caught by, carried, and thrown from a net on a stick.

Albuminous

$\begin{array}{ll}\text { Axial } & \text { Alchemy } \\ \text { Buffoonery } & \text { Bibliophile } \\ \text { Baulk } & \text { Braise }\end{array}$

Closure

Disencumber

Dissociation

Hemispherical

Informality

Lethal

Lacrosse

Cassock

Determinist

Diminuendo

Idiomatic

\section{Semantically Related Interlopers}

Adherent of the view that whether God exists is unknown.

Obvious or trite.

Female spirit whose wail portends death.

The complete remains of a dead animal, especially at a butcher's.

Stoppered glass vessel in which spirits are brought to the table.

Someone who listens to other people's conversations.

Very small in size, as in Gulliver's Travels.

The desire to do evil, or for others to have ill fortune.

Saying little, reserved, uncommunicative.

Hollow or, as regards people, unintelligent, expressionless.

\section{Evangelism}

Uninspired

Incubus

Disinter

Carafe

Sleuth

Teeny

Acrimonious

Awestruck

Abnegation
Iniquitous

Literate

Hypochondriac

Agnostic

Banal

Banshee

Carcass

Decanter

Eavesdropper

Lilliputian

Malevolence

Taciturn

Vacuous 
APPENDIX (Continued)

\begin{tabular}{|c|c|c|}
\hline Definition & Interloper & Target \\
\hline \multicolumn{3}{|c|}{ Unrelated Interlopers } \\
\hline Like a beautiful, angelic child. & Opinionated & Cherubic \\
\hline $\begin{array}{l}\text { The study of the developing foetus before } \\
\text { birth. }\end{array}$ & Wholehearted & Embryology \\
\hline $\begin{array}{l}\text { House of rest for travelers or for the } \\
\text { terminally ill, often kept by a } \\
\text { religious order. }\end{array}$ & Fossilize & Hospice \\
\hline To keep eggs warm until hatching. & Simulation & Incubate \\
\hline $\begin{array}{l}\text { Puzzle in which one tries to piece } \\
\text { together a broken picture. }\end{array}$ & Tyrannize & Jigsaw \\
\hline $\begin{array}{l}\text { Term describing an issue that is open to } \\
\text { discussion or modification. }\end{array}$ & Scalpel & Negotiable \\
\hline Relating to the great seas of the world. & Naturalism & Oceanic \\
\hline Feeding on both plants and flesh. & Unavailable & Omniverous \\
\hline $\begin{array}{l}\text { To make calm or serene or to reduce } \\
\text { agitation, especially by use of a drug. }\end{array}$ & Stepson & Tranquillize \\
\hline $\begin{array}{l}\text { Common term for injury to the neck } \\
\text { caused by a sudden jerk of the } \\
\text { head, for example in vehicle collision. }\end{array}$ & Cinnabar & Whiplash \\
\hline
\end{tabular}

(Manuscript received March 30, 1987;

revision accepted for publication April 7, 1988.) 